OPEN ACCESS

Edited by:

Tony Jourdan,

U1231 Lipides, Nutrition, Cancer (LNC) (INSERM), France

Reviewed by: Alessandra Peres,

Federal University of Health Sciences of Porto Alegre, Brazil Jonathan Peter Little,

University of British Columbia, Canada

*Correspondence:

Dante S. Harbuwono

dante.saksono@ui.ac.id orcid.org/0000-0002-6006-2719

Specialty section: This article was submitted to

Diabetes: Molecular Mechanisms, a section of the journal

Frontiers in Endocrinology

Received: 13 January 2021 Accepted: 13 July 2021 Published: 29 July 2021

Citation:

Wibowo H, Harbuwono DS, Tahapary DL, Kartika R, Pradipta S and Larasati RA (2021) Impact of Sodium Butyrate Treatment in LPS-

Stimulated Peripheral Blood

Mononuclear Cells of Poorly

Controlled Type 2 DM.

Front. Endocrinol. 12:652942. doi: 10.3389/fendo.2021.652942

\section{Impact of Sodium Butyrate Treatment in LPS-Stimulated Peripheral Blood Mononuclear Cells of Poorly Controlled Type 2 DM}

\author{
Heri Wibowo ${ }^{1}$, Dante S. Harbuwono ${ }^{2 *}$, Dicky L. Tahapary ${ }^{2}$, Rona Kartika ${ }^{2}$, \\ Saraswati Pradipta ${ }^{3}$ and Rahma A. Larasati ${ }^{4}$
}

${ }^{1}$ Department of Parasitology, Faculty of Medicine, Universitas Indonesia, Jakarta, Indonesia, ${ }^{2}$ Division of Metabolic Endocrinology and Diabetes, Department of Internal Medicine, Faculty of Medicine, Universitas Indonesia, Jakarta, Indonesia, ${ }^{3}$ Integrated Laboratory, Faculty of Medicine, Universitas Indonesia, Jakarta, Indonesia, ${ }^{4}$ Department of Biomedicines, Faculty of Medicine, Universitas Muhammadiyah Jakarta, Jakarta, Indonesia

Type 2 diabetes mellitus (T2DM) is associated with chronic low-grade inflammation, which is marked by the dysregulation of innate and adaptive immune responses. Therefore, reducing inflammation, possibly through an immunoregulatory agent, may play a role in T2DM treatment. Butyrate is the most potent short-chain fatty acid (SCFA), and it exerts anti-inflammatory properties by inhibiting histone deacetylase activity. As an immunoregulatory agent, sodium butyrate can inhibit nuclear factor kB (NF-kB) activation and reduce the production of pro-inflammatory cytokines in immune cells. The aim of the study was to measure the level of plasma butyrate in poorly controlled T2DM and normoglycemic participants and to compare the response of peripheral blood mononuclear cells (PBMCs) to sodium butyrate treatment between the groups by measuring production of the following cytokines: tumor necrosis factor (TNF)- $\alpha$, interleukin (IL)-6, interferon (IFN)- $\gamma, \mathrm{IL}-13$, and IL-10. The in vitro study examined the PBMCs of 15 participants with poorly controlled T2DM and 15 normoglycemic participants. PBMCs were cultured with the following stimulations for two days at a temperature of $37^{\circ} \mathrm{C}$ and $5 \% \mathrm{CO}_{2}: 100 \mathrm{ng} / \mathrm{mL}$ lipopolysaccharide (LPS), $1 \mathrm{mM}$ sodium butyrate, or a combination of $100 \mathrm{ng} / \mathrm{mL}$ LPS and $1 \mathrm{mM}$ sodium butyrate. Plasma butyrate was measured using gas chromatography-mass spectrometry, and cytokines from culture supernatant were analyzed using magnetic beads multiplex assay. Plasma butyrate levels in participants with poorly controlled T2DM did not significantly differ from those in normoglycemic participants $(p=0.105)$. Compared to treatment with an LPSstimulated PBMC culture, treatment with $1 \mathrm{mM}$ sodium butyrate reduced the levels of TNF- $\alpha(p<0.039)$ and IFN- $\gamma(p<0.038)$ in normoglycemic participants. The same general trend was seen in PBMC from participants with poorly controlled T2DM, but higher variability appeared to preclude statistical significance. These data suggest that butyrate 
may modulate inflammatory cytokine production in human PBMCs, but more research is needed to determine if butyrate is anti-inflammatory in poorly controlled T2DM.

Keywords: poorly controlled type 2 diabetes mellitus, inflammatory response, peripheral blood mononuclear cells, butyrate, lipopolysaccharide (LPS)

\section{INTRODUCTION}

Gut microbiota have an essential function in maintaining intestinal homeostasis and human health by regulating the immune system, maintaining epithelial barriers, and protecting against several diseases (1). Among the numerous metabolites produced by commensal gut bacteria, short-chain fatty acids (SCFAs) have received the most attention in the context of alleviating host health conditions. SCFAs are the end products of fermentation contained carbolic acids with aliphatic tails. SCFAs are found in high concentrations in the gut lumen, and they can be absorbed via non-ionic diffusion across colonic epithelial cells (2).

In addition to being the major energy source for colonocytes (3), SCFAs have long been known to modulate the immune response. Among SCFAs, butyrate is the main type that exerts anti-inflammatory properties (4). Butyrate affects neutrophil function and migration (5) and inhibits nuclear factor kappa B $(\mathrm{NF}-\mathrm{\kappa B})$, tumor necrosis factor (TNF)- $\alpha$, and interleukin (IL)- $1 \beta$ (6). In addition, butyrate reduces the adherence of monocytes or lymphocytes to cytokine-stimulated endothelial cells (7) and inhibits interferon- $\boldsymbol{\gamma}$ signaling (8). Butyrate probably regulates the immune system by modifying cellular processes, such as promoting the activation of $\mathrm{G}$-protein coupled receptors (GPCRs), inhibiting histone deacetylase (HDAC), and stimulating histone acetyltransferase (4). Correa-Oliveira et al. (4) demonstrated that butyrate could hinder the development of murine bone marrow-derived macrophages, suppress the activation of T cells, and induce apoptosis. In Crohn's disease, butyrate has been known to reduce TNF production and inhibit $\mathrm{NF}-\kappa \mathrm{B}$ activation in lamina propria cells and peripheral blood mononuclear cells (PBMCs) (9).

Type 2 diabetes mellitus (T2DM) is a metabolic disease characterized by hyperglycemia and insulin resistance $(10,11)$ and is often associated with low-grade inflammatory conditions (12). In addition, T2DM is correlated with adaptive immune dysfunction, which is assessed by $\mathrm{T}$ helper (Th)-1/Th-2 imbalance (12). Our previous study showed that PBMCs from T2DM patients demonstrated an enhanced cellular responsiveness to phytohemagglutinin (PHA), marked by lower interferon (IFN)- $\gamma$ production, lower indoleamine 2,3 dioxygenase production, and higher TNF- $\alpha / \mathrm{IFN}-\gamma$ and IL-6/ IFN- $\gamma$ ratios compared to normoglycemic participants $(13,14)$.

Treatment with SCFAs, particularly butyrate, is thought to be beneficial for patients with low-grade inflammatory conditions such as T2DM, but data concerning the effect of butyrate on reducing cytokine production from PBMC in T2DM patients are limited. Sodium butyrate was found to ameliorate insulin resistance and decrease plasma glucose levels in diabetic rats (15). In addition, Larasari et al. (16) showed that the short-term butyrate treatment reduced the accumulated distance of monocyte migration and decreased the inflammatory potential released by monocytes in healthy subjects and in T2DM patients. In the current study, participants' levels of plasma butyrate were measured, and the responses of peripheral blood mononuclear cells (PBMCs) to sodium butyrate were compared between poorly controlled T2DM patients and normoglycemic participants in the presence of lipopolysaccharide; this comparison was made by measuring cytokine production from innate immunity (TNF- $\alpha$ and IL-6) and Th1/Th2 cytokine balance (IFN- $\gamma$, IL-13, and IL-10).

\section{MATERIALS AND METHODS}

\section{Participants}

The in vitro study was performed on PBMCs collected from 15 normoglycemic participants and 15 participants with poorly controlled T2DM. In our former study of T2DM, the sample size of participants with T2DM was 21 people. In this study, we excluded six of those participants, whose T2DM was controlled. Therefore, only 15 participants were included in this analysis.

The normoglycemic participants were 30-55-year-olds with fasting blood glucose $(\mathrm{FBG})<100 \mathrm{mg} / \mathrm{dL}$ and glycated hemoglobin $(\mathrm{HbA1c})<5.7 \%$ who had never been diagnosed with T2DM by a physician. The criteria for poorly controlled T2DM patients, according to a glycemic target from the American Diabetes Association (17), are people 30-55 years of age, with $\mathrm{FBG}>130 \mathrm{mg} / \mathrm{dL}$, and/or $\mathrm{HbAlc}>7 \%$. The exclusion criteria for both groups were current pregnancy, steroid use, use of non-steroidal anti-inflammatory drugs or antibiotics within the two weeks preceding the study, and infection at the time of blood collection, including symptoms of fever, sore throat, respiratory tract infection, or urinary tract infection.

The study was approved by the ethical committee of the Faculty of Medicine of Universitas Indonesia, reference number 18-03-0236. All participants provided their written informed consent prior to taking part in this research.

\section{Plasma Butyrate Analysis}

Plasma butyrate was analyzed using Agilent 5973 mass spectrometry with 6890 Plus gas chromatography system (Agilent Technologies, Santa Clara, CA, USA). In brief, $1 \mathrm{~mL}$ of plasma was added to $3 \mathrm{~mL}$ of $5 \%$ formic acid and $2.5 \mathrm{~mL}$ of ethyl acetate. The suspension was homogenized for 30 minutes and centrifuged at $15000 \mathrm{rpm}$ for 5 minutes. Six $\mathrm{mL}$ of supernatant was isolated, and $0.1 \mathrm{~g}$ sodium sulfate anhydrous was added. Then, the solution was injected into the gas chromatography-mass spectrometry machine. 


\section{Peripheral Blood Mononuclear Cells (PBMC) Isolation and Culture}

PBMCs were isolated by centrifugation from Roswell Park Memorial Institute (RPMI) 1640-diluted blood through FicollPaque Plus (GE Healthcare Life Sciences, Chicago, IL, USA). Then, PBMCs were resuspended in a cell culture medium consisting of RPMI 1640 supplemented with 10\% heatinactivated fetal bovine serum and $1 \%$ penicillin-streptomycin at a concentration of $10^{6}$ cells $/ \mathrm{mL}$. A $500 \mu \mathrm{L}$ cell suspension was incubated in a 24-well tissue culture plate for one day at a temperature of $37^{\circ} \mathrm{C}$ with $5 \% \mathrm{CO}$. Then, either $500 \mu \mathrm{L}$ of 200 $\mathrm{ng} / \mathrm{mL}$ LPS (Sigma Aldrich, St. Louis, MO, USA), $500 \mu \mathrm{L}$ of $2 \mathrm{mM}$ sodium butyrate (Sigma Aldrich), $500 \mu \mathrm{L}$ of $200 \mathrm{ng} / \mathrm{mL}$ LPS and $2 \mathrm{mM}$ sodium butyrate, or $500 \mu \mathrm{L}$ of cell culture medium (the unstimulated group) were added to the wells. Then, the culture plates were incubated for $48 \mathrm{~h}$ at a temperature of $37^{\circ} \mathrm{C}$ with $5 \% \mathrm{CO} 2$. On day three, cell culture supernatants were harvested and stored at a temperature of $-80^{\circ} \mathrm{C}$.

\section{Cytokines Assay}

Cell culture supernatants were measured for in vitro cytokine production. TNF- $\alpha$, IL-6, IFN- $\gamma$, IL-13, and IL-10 were measured using bead-based multiplex assay (R\&D Systems, Minneapolis, MN, USA) as described in a previous paper (13). The detection limits were $0.6 \mathrm{pg} / \mathrm{mL}$ for TNF- $\alpha ; 1.11 \mathrm{pg} / \mathrm{mL}$ for interleukin- 6 (IL-6); $1.27 \mathrm{pg} / \mathrm{mL}$ for interferon- $\gamma$ (IFN- $\gamma$ ); $2.01 \mathrm{pg} / \mathrm{mL}$ for interleukin-13 (IL-13); and $0.3 \mathrm{pg} / \mathrm{mL}$ for interleukin-10 (IL-10).

\section{Data Analysis}

Data were analyzed using IBM SPSS Statistics, Version 23.0 (IBM, Armonk, NY, USA). A Shapiro-Wilk test was used to assess normality between groups. Since the baseline characteristics were normally distributed, unpaired Student's $t$ tests were used to compare the baseline characteristics of the two groups. Plasma butyrate concentration and cytokine production were not normally distributed. Therefore, the Mann-Whitney $U$ test was used to compare the plasma butyrate concentrations of the two groups, and the Kruskal-Wallis test followed by Bonferroni adjustment were used to conduct six comparisons of the differences in cytokine production among the four stimulation groups: LPS, LPS and butyrate, butyrate, and unstimulated culture. Because they were not normally distributed, data of plasma butyrate concentration and cytokines' production were presented as medians (minimummaximum). GraphPad Prism 8 was used to create graphs and data visualization. The data were considered statistically significant if the $p$-value was $<0.05$.

\section{RESULTS}

\section{Subject Characteristics}

The baseline characteristics of the 15 normoglycemic participants and 15 participants with poorly controlled T2DM enrolled in this study are presented in Table 1. As expected, the
TABLE 1 | Baseline subject characteristics.

\begin{tabular}{|c|c|c|c|}
\hline $\begin{array}{l}\text { Baseline subject } \\
\text { characteristics }\end{array}$ & $\begin{array}{l}\text { Normoglycemic } \\
\text { group }(n=15)\end{array}$ & $\begin{array}{l}\text { Poorly controlled } \\
\text { T2DM group }(n=15)\end{array}$ & $p$-value \\
\hline Female sex $(n, \%)$ & $10(66.7)$ & $8(55.6)$ & 0.355 \\
\hline Male sex (n, \%) & 5 (33.3) & $7(44.4)$ & \\
\hline Age (years) & $39.73 \pm 4.52$ & $47.33 \pm 6.55$ & $0.001^{\star \star}$ \\
\hline BMI $\left(\mathrm{kg} / \mathrm{m}^{2}\right)$ & $22.81 \pm 2.78$ & $26.80 \pm 2.81$ & $0.001^{\star \star}$ \\
\hline FBG (mg/dL) & $89.51 \pm 11.84$ & $205.53 \pm 99.59$ & $<0.001^{\star \star \star \star}$ \\
\hline HbA1c (\%) & $4.87 \pm 0.52$ & $9.38 \pm 2.95$ & $<0.001^{\star \star \star}$ \\
\hline
\end{tabular}

T2DM, type 2 diabetes mellitus; BMI, body mass index; FBG, fasting blood glucose; HDA1c, glycosylated hemoglobin; ${ }^{* *} p<0.01,{ }^{* * *} p<0.001$. Age, BMI, FBG, and HbA1C were presented as mean \pm standard deviation, and statistical significances were calculated using unpaired Student's t-tests. The percentage of participants of each sex was calculated using Fisher's exact test.

poorly controlled T2DM group had higher FBG and HbA1c with a mean \pm standard deviation of $205.53 \pm 99.59 \mathrm{mg} / \mathrm{dL}$ and $9.38 \pm$ $2.95 \%$, respectively. Since this study did not match the age and body mass index (BMI) of participants, the normoglycemic group was younger and leaner than the T2DM group, which introduced a high chance of confounding factors; thus, we included age and BMI as covariates for further analysis.

\section{Plasma Butyrate in T2DM Patients}

As shown in Figure 1, even after age and BMI adjustment, plasma butyrate levels of participants with poorly controlled T2DM did not significantly differ from normoglycemic participants [median $0.24(0.14-0.78)$ vs $0.18(0.12-0.69)$ $\mathrm{mM}, p=0.105]$.

\section{Cytokine Concentration in PBMC Culture Supernatants}

According to the Kruskal-Wallis test (Table 2), the production of TNF- $\alpha$, IL-6, IFN- $\gamma$, and IL-10 were different in participants with poorly controlled T2DM and normoglycemic participants. However, there was no difference in the cytokine production of PBMC cultures between poorly controlled T2DM and normoglycemic participants (Supplemental Table 1). Post hoc testing found that TNF- $\alpha$, IL- 6 , IFN- $\gamma$, and IL-10 were more greatly increased with LPS stimulation alone compared to butyrate stimulation and no stimulation in both normoglycemic and poorly controlled T2DM participants (Figure 2).

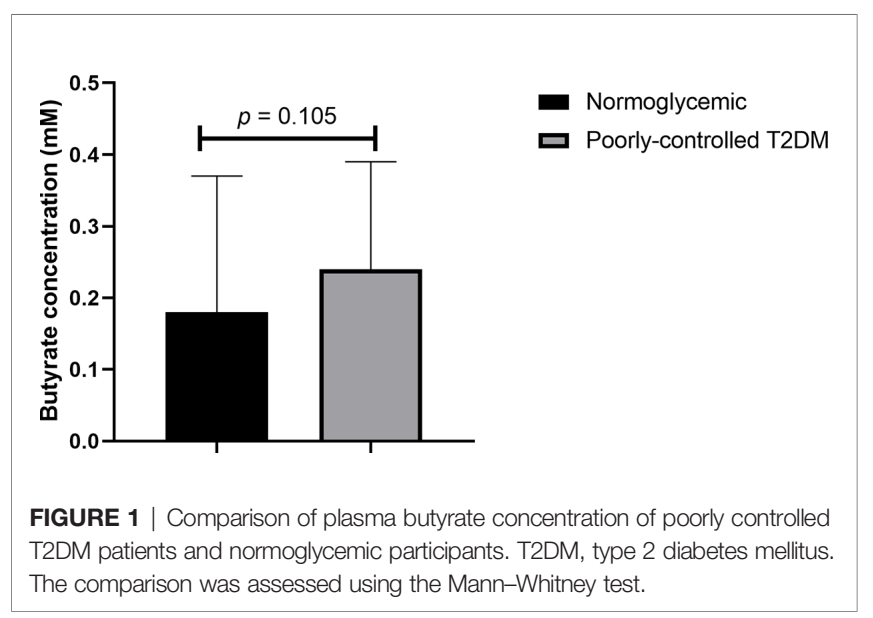


TABLE 2 | Kruskal-Wallis test to compare the cytokine production of various stimulated PBMC cultures in the study groups.

\begin{tabular}{|c|c|c|c|c|c|c|}
\hline Cytokines & Stimulants & $\mathbf{n}$ & Median (minimum-maximum) & H statistics & df & $p$-value \\
\hline \multicolumn{7}{|c|}{ Normoglycemic subject } \\
\hline \multirow[t]{4}{*}{$\mathrm{TNF}-\alpha$} & LPS & 15 & 707 (73 - 4071) & 45.832 & 3 & $<0.001^{\star \star \star}$ \\
\hline & LPS + Butyrate & 15 & $104(2-743)$ & & & \\
\hline & Butyrate & 15 & $4(0.6-15)$ & & & \\
\hline & Unstimulated & 15 & $2(1-8)$ & & & \\
\hline \multirow[t]{4}{*}{ IL-6 } & LPS & 15 & $2285.32(373.66-8034.47)$ & 42.013 & 3 & $<0.001^{\star \star \star}$ \\
\hline & LPS + Butyrate & 15 & $1618(8.85-6044.9)$ & & & \\
\hline & Butyrate & 15 & $49(7-184.15)$ & & & \\
\hline & Unstimulated & 15 & $20.724(3.55-79.38)$ & & & \\
\hline \multirow[t]{4}{*}{ Interferon- $\gamma$} & LPS & 15 & $31.52(6.12-584.63)$ & 20.018 & 3 & $<0.001^{\star \star \star}$ \\
\hline & LPS + Butyrate & 15 & $13.93(6.12-29.57)$ & & & \\
\hline & Butyrate & 15 & $8.07(1.27-25.66)$ & & & \\
\hline & Unstimulated & 15 & $10.02(1.27-19.80)$ & & & \\
\hline \multirow[t]{4}{*}{$\| \mathrm{L}-13$} & LPS & 15 & $254.381(12.44-625.13)$ & 4.511 & 3 & 0.211 \\
\hline & LPS + Butyrate & 15 & $66.286(8.26-328.18)$ & & & \\
\hline & Butyrate & 15 & $61.194(8.25-454.08)$ & & & \\
\hline & Unstimulated & 15 & $61.194(12.44-259.42)$ & & & \\
\hline \multirow[t]{4}{*}{ IL-10 } & LPS & 15 & $50.909(15.85-365.6)$ & 42.247 & 3 & $<0.001^{\star \star \star}$ \\
\hline & LPS + Butyrate & 15 & $19(4.46-59.49)$ & & & \\
\hline & Butyrate & 15 & $3(0.3-4.38)$ & & & \\
\hline & Unstimulated & 15 & $3.62(0.3-24.09)$ & & & \\
\hline \multicolumn{7}{|c|}{ Poorly Controlled Type 2 Diabetes Mellitus } \\
\hline \multirow[t]{4}{*}{$\mathrm{TNF}-\alpha$} & LPS & 15 & $1030(301-4235)$ & 47.725 & 3 & $<0.001^{\star \star \star}$ \\
\hline & LPS + Butyrate & 15 & $208(27-2461)$ & & & \\
\hline & Butyrate & 15 & $3(1-34)$ & & & \\
\hline & Unstimulated & 15 & $3(1-7)$ & & & \\
\hline \multirow[t]{4}{*}{ IL-6 } & LPS & 15 & 3185.405 (1215.6 - 7591.23) & 47.010 & 3 & $<0.001^{\star \star \star}$ \\
\hline & LPS + Butyrate & 15 & $1299.974(189.2-16554.46)$ & & & \\
\hline & Butyrate & 15 & $36.752(10.44-351.70)$ & & & \\
\hline & Unstimulated & 15 & $21.737(3.73-110.93)$ & & & \\
\hline \multirow[t]{4}{*}{ Interferon- $\gamma$} & LPS & 15 & $33.48(8.07-178.11)$ & 19.716 & 3 & $<0.001^{\star \star \star}$ \\
\hline & LPS + Butyrate & 15 & $14.19(1.27-295.37)$ & & & \\
\hline & Butyrate & 15 & $8.07(1.27-19.80)$ & & & \\
\hline & Unstimulated & 15 & $11(1.27-28.89)$ & & & \\
\hline \multirow[t]{4}{*}{ IL-13 } & LPS & 15 & $329.319(24.78-731.42)$ & 7.287 & 3 & 0.063 \\
\hline & LPS + Butyrate & 15 & $121.426(16.52-517.07)$ & & & \\
\hline & Butyrate & 15 & $121.426(8.26-675)$ & & & \\
\hline & Unstimulated & 15 & $97.505(8.26-315.86)$ & & & \\
\hline \multirow[t]{4}{*}{ IL-10 } & LPS & 15 & $60.931(14.55-258.00)$ & 45.945 & 3 & $<0.001^{\star \star \star}$ \\
\hline & LPS + Butyrate & 15 & $22.437(8.45-67.89)$ & & & \\
\hline & Butyrate & 15 & $2.838(0.3-5.62)$ & & & \\
\hline & Unstimulated & 15 & $2.208(0.3-16.75)$ & & & \\
\hline
\end{tabular}

TNF- $\alpha$, tumor necrosis factor- $\alpha$; IL-6, interleukin-6; IL-13, interleukin-13; IL-10, interleukin 10; LPS, lipopolysaccharide at total concentration of 100 ng/mL; LPS + butyrate, lipopolysaccharide at total concentration of $100 \mathrm{ng} / \mathrm{mL}$ and sodium butyrate at total concentration of $1 \mathrm{mM}$; and butyrate, sodium butyrate stimulation at total concentration of $1 \mathrm{mM}$; $d f$, degree of freedom. The comparisons were assessed using the Kruskal-Wallis test. The data are presented as median (minimum-maximum) pg/mL. ${ }^{* *} p<0.001$.

Compared to PBMC culture stimulated with LPS alone, treatment with sodium butyrate in LPS-stimulated culture suppressed only pro-inflammatory cytokines-TNF- $\alpha$ $(p=0.039)$ and IFN- $\gamma(p=0.038)$-in normoglycemic participants. Interestingly, although cytokine production from LPS stimulated culture in participants with poorly controlled T2DM was similar to that of normoglycemic participants, treatment with $1 \mathrm{mM}$ sodium butyrate did not significantly reduce TNF- $\alpha(p=0.062)$ or IFN- $\gamma(p=0.065)$ production.

Without LPS stimulation, treatment with sodium butyrate was unable to stimulate or suppress PBMC culture, which was shown by the similar amount of cytokine production in the unstimulated PBMC culture and the culture stimulated with sodium butyrate alone (Figure 2). Moreover, sodium butyrate treatment did not affect the production of IL-6, IL-13, and IL-10 by LPS-stimulated culture either in the normoglycemic or poorly controlled T2DM group (Figures 2B, D, E).

\section{DISCUSSION}

T2DM is classified as a low-grade inflammatory condition (12). Low-grade inflammatory condition is closely related to altered intestinal microbiota or dysbiosis $(18,19)$. Dysbiosis alters SCFA production in T2DM, thus making the body vulnerable to inflammation (20). Moreover, several studies have revealed that translocation of gut microbiota, especially endotoxins and LPS, worsens inflammation in $\operatorname{T2DM}(21,22)$.

Butyrate is the most potent SCFA that exerts antiinflammatory properties. About $95 \%$ of butyrate is absorbed 


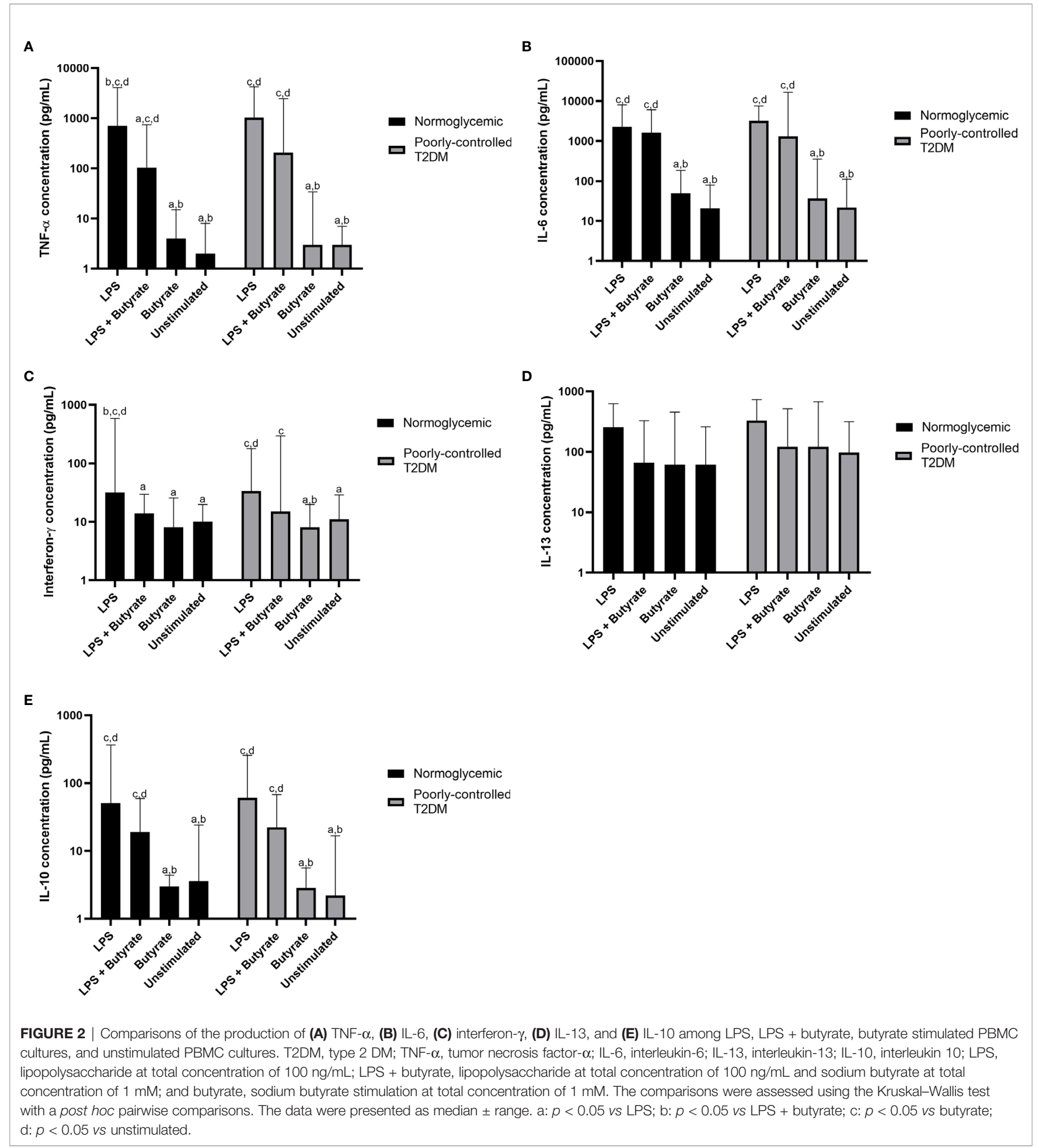

from the intestinal lumen (23). However, the plasma butyrate level is far lower than the intracolonic butyrate level because colonocytes use butyrate as an energy source. In our study, the plasma butyrate concentration was in the range of $0.1-0.8 \mathrm{mM}$ in participants with poorly controlled T2DM and $0.1-0.7 \mathrm{mM}$ in normoglycemic participants. These concentrations are higher than that of healthy normoglycemic western population, which has a concentration in the range of 0.01-0.1 $\mathrm{mM}(24,25)$. This finding might be influenced by the Asian diet, which is relatively low in meat, dairy products, and sugar but high in vegetables and resistant starches. We also found that plasma butyrate did not differ between poorly controlled T2DM and normoglycemic 
participants. Contrary to our finding, Muller et al. (26) showed that fasting circulating butyrate was negatively associated with fasting glucose and free fatty acid levels but was not correlated with inflammatory markers in obese, non-diabetes participants. Nishitsuji et al. (20) also found that although total SCFA levels were lower in obese diabetic mice than in non-obese mice, the plasma butyrate levels of obese diabetic mice were higher, which was correlated with an increased ratio of Gram-positive to Gramnegative gut microbiota.

Nancey et al. (27) reported that the anti-inflammatory effect of butyrate was achieved when butyrate was given in concentrations higher than the physiological level. In our study, we cultured PBMC in the presence of $1 \mathrm{mM}$ sodium butyrate. In normoglycemic participants, sodium butyrate significantly reduced the LPS-stimulated release of TNF- $\alpha$ but not IL-6. Segain et al. (9) reported that the LPS-induced translocation of NF-kB was inhibited by sodium butyrate. Therefore, butyrate treatment downregulated the LPSstimulated mRNA expression of TNF- $\alpha$, TNF- $\beta$, IL- 6 , and IL$1 \beta$. Similarly to our result, Nancey et al. (27) demonstrated that although TNF- $\alpha$ and IL- 6 were encoded by the same transcription factor, NF-kB, sodium butyrate $(0.0625-2 \mathrm{mM})$ was found to inhibit the LPS + PHA-stimulated release of TNF$\alpha$ but not IL-6. Moreover, butyrate did not affect IL- 6 production induced by Mycobacterium tuberculosis (28). Butyrate also had an inhibitory effect on Th-1 cell activity, marked by a reduced release of IFN- $\gamma$ in normoglycemic subjects, but sodium butyrate did not affect the anti-inflammatory activity of Th-2 cytokines (IL-13 and IL-10) in either group. In line with this result, and despite the possibility that IL-10 would act as a mediator of butyrate's anti-inflammatory effect, Lachmandas et al. (28) reported that SCFAs, including butyrate, also failed to affect LPS-induced IL-10 production. The presence of such conflicting results even in normoglycemic or healthy participants suggests that the mechanism by which sodium butyrate affects cytokine production is complex and warrants further study.

Interestingly, in the PBMC of participants with poorly controlled T2DM, $1 \mathrm{mM}$ butyrate treatment did not affect LPS-stimulated cytokine release, either innate or adaptive, in contrast to findings in normoglycemic participants. Cleophas et al. (29) found that four-week oral sodium butyrate supplementation in participants with metabolic syndrome showed that oral sodium butyrate did not influence the cytokine-producing capacity of PBMC assessed by measuring IL-1 $\beta$, IL-1Ra, IL-6, and IL-10 upon bacterial stimulation. We can only speculate that our sodium butyrate concentration was inadequate to inhibit HDAC activity or that PBMCs from participants with poorly controlled T2DM have an impaired response to sodium butyrate treatment. The failure of sodium butyrate treatment was also seen in ulcerative colitis patients: Magnusson et al. (30) reported that sodium butyrate was more potent in down-regulating inflammatory gene expression in noninflamed healthy tissue than in the inflamed tissue of ulcerative colitis patients.

Some limitations of our study need to be addressed. First, we studied the effects of a single concentration of sodium butyrate on
LPS-stimulated cytokine release in PBMCs. As the $1 \mathrm{mM}$ sodium butyrate only partly affected cytokine release in normoglycemic and poorly controlled T2DM participants, gene expression and the dose-effect relationship should be studied to elaborate our findings. Second, we did not compare the plasma butyrate and cytokine production of participants with well-controlled T2DM to those with metabolic syndrome to investigate the time when the cytokine-producing capacity of PBMC is impaired. Third, these results do not reflect the in vivo condition in participants with poorly controlled T2DM because the duration of diabetes, micro- and macrovascular complications, and various diabetes medications could interfere with the intestinal microbiota and immune responses. However, these findings may at least partly explain why high plasma butyrate levels are not adequate to maintain an anti-inflammatory response in T2DM, particularly in poorly controlled T2DM.

\section{CONCLUSION}

Plasma butyrate levels in participants with poorly controlled T2DM were not significantly different from those in normoglycemic participants. Compared to treatment with an LPS-stimulated PBMC culture, treatment with $1 \mathrm{mM}$ sodium butyrate reduced the levels of TNF- $\alpha(\mathrm{p}<0.039)$ and IFN- $\gamma(\mathrm{p}<$ 0.038 ) in normoglycemic participants. The same general trend was seen in PBMC from participants with poorly controlled T2DM, but higher variability appeared to preclude statistical significance. These data suggest that sodium butyrate may modulate inflammatory cytokine production in human PBMCs but more research is needed to determine if butyrate is antiinflammatory in poorly controlled T2DM.

\section{DATA AVAILABILITY STATEMENT}

The raw data supporting the conclusions of this article will be made available by the authors, without undue reservation.

\section{ETHICS STATEMENT}

The studies involving human participants were reviewed and approved by the ethical committee of the Faculty of Medicine from Universitas Indonesia with reference number 18-03-0236. The patients/participants provided their written informed consent to participate in this study.

\section{AUTHOR CONTRIBUTIONS}

HW and DS contributed to conception, design, and acquisition; critically drafted the manuscript; revised the manuscript; gave final approval; and agreed to be accountable for all aspects of work ensuring integrity and accuracy. DT and RK contributed to acquisition, critically drafted the manuscript, revised the 
manuscript, gave final approval, and agreed to be accountable for all aspects of work ensuring integrity and accuracy. SP and RL contributed to interpretation, critically revised the manuscript, gave final approval, and agreed to be accountable for all aspects of work ensuring integrity and accuracy. All authors contributed to the article and approved the submitted version.

\section{FUNDING}

This research was supported by Universitas Indonesia Research Grant (PUTI) Q2 with reference number NKB-4084/UN2.RST/ HKP.05.00/2020.

\section{ACKNOWLEDGMENTS}

The authors would like to thank Dr. Ekowati Rahajeng, and the participants, nurses, and doctors involved in this cohort study for

\section{REFERENCES}

1. Baxter NT, Schmidt AW, Venkataraman A, Kim KS, Waldron C, Schmidt TM. Dynamics of Human Gut Microbiota and Short-Chain Fatty Acids in Response to Dietary Interventions With Three Fermentable Fibers. mBio (2019) 10(1):1-13. doi: 10.1128/mBio.02566-18

2. McNabney SM, Henagan TM. Short Chain Fatty Acids in the Colon and Peripheral Tissues: A Focus on Butyrate, Colon Cancer, Obesity and Insulin Resistance. Nutrients (2017) 9(12):1348. doi: 10.3390/nu9121348

3. Parada Venegas D, de la Fuente MK, Landskron G, González MJ, Quera R, Dijkstra G, et al. Short Chain Fatty Acids (Scfas)-Mediated Gut Epithelial and Immune Regulation and Its Relevance for Inflammatory Bowel Diseases. Front Immunol (2019) 10(277):1-16. doi: 10.3389/fimmu.2019.00277

4. Corrêa-Oliveira R, Fachi JL, Vieira A, Sato FT, Vinolo MA. Regulation of Immune Cell Function by Short-Chain Fatty Acids. Clin Transl Immunol (2016) 5(4):e73. doi: 10.1038/cti.2016.17

5. Böcker U, Nebe T, Herweck F, Holt L, Panja A, Jobin C, et al. Butyrate Modulates Intestinal Epithelial Cell-Mediated Neutrophil Migration. Clin Exp Immunol (2003) 131(1):53-60. doi: 10.1046/j.1365-2249.2003. 02056.x

6. Zapolska-Downar D, Siennicka A, Kaczmarczyk M, Kołodziej B, Naruszewicz M. Butyrate Inhibits Cytokine-Induced VCAM-1 and ICAM-1 Expression in Cultured Endothelial Cells: The Role of NF-kappaB and Pparalpha. J Nutr Biochem (2004) 15(4):220-8. doi: 10.1016/j.jnutbio.2003.11.008

7. Menzel T, Lührs H, Zirlik S, Schauber J, Kudlich T, Gerke T, et al. Butyrate Inhibits Leukocyte Adhesion to Endothelial Cells Via Modulation of VCAM1. Inflammation Bowel Dis (2004) 10(2):122-8. doi: 10.1097/00054725200403000-00010

8. Klampfer L, Huang J, Sasazuki T, Shirasawa S, Augenlicht L. Inhibition of Interferon Gamma Signaling by the Short Chain Fatty Acid Butyrate. Mol Cancer Res (2003) 1(11):855-62.

9. Segain JP, de la Blétière DR, Bourreille A, Leray V, Gervois N, Rosales C, et al. Butyrate Inhibits Inflammatory Responses Through NFkappaB Inhibition: Implications for Crohn's Disease. Gut (2000) 47(3):397-403. doi: 10.1136/ gut.47.3.397

10. Kim CH. Microbiota or Short-Chain Fatty Acids: Which Regulates Diabetes? Cell Mol Immunol (2018) 15(2):88-91. doi: 10.1038/cmi.2017.57

11. Mandaliya DK, Seshadri S. Short Chain Fatty Acids, Pancreatic Dysfunction and Type 2 Diabetes. Pancreatology (2019) 19(2):280-4. doi: 10.1016/ j.pan.2019.01.021

12. Chen L, Chen R, Wang H, Liang F. Mechanisms Linking Inflammation to Insulin Resistance. Int J Endocrinol (2015) 2015:508409. doi: 10.1155/2015/ 508409 non-communicable disease. Also, we want to express our appreciation to the National Institute of Health Research and Development, the Ministry of Health, Indonesia, all the staff at the Integrated Laboratory Faculty of Medicine, Universitas Indonesia, and all the others who contributed to this research. This article was presented at the 5th International Conference and Exhibition on Indonesian Medical Education and Research Institute (5th ICE on IMERI), Faculty of Medicine, Universitas Indonesia. We thank the 5th ICE on IMERI Committee, who had supported the peer review and manuscript preparation before submitting it to the journal.

\section{SUPPLEMENTARY MATERIAL}

The Supplementary Material for this article can be found online at: https://www.frontiersin.org/articles/10.3389/fendo.2021. 652942/full\#supplementary-material

13. Kartika R, Purnamasari D, Pradipta S, Larasati RA, Wibowo H. Impact of Low Interferon- $\gamma$ and IL-10 Levels on TNF- $\alpha$ and IL-6 Production by PHAInduced Pbmcs in Type 2 Diabetes Mellitus. J Inflamm Res (2020) 13:187-93. doi: 10.2147/JIR.S245064

14. Kartika R, Wibowo H, Purnamasari D, Pradipta S, Larasati RA. Altered Indoleamine 2,3-Dioxygenase Production and Its Association to Inflammatory Cytokines in Peripheral Blood Mononuclear Cells Culture of Type 2 Diabetes Mellitus. Int J Tryptophan Res (2020) 13:1178646920978236. doi: $10.1177 / 1178646920978236$

15. Khan S, Jena G. Sodium Butyrate Reduces Insulin-Resistance, Fat Accumulation and Dyslipidemia in Type-2 Diabetic Rat: A Comparative Study With Metformin. Chem Biol Interact (2016) 254:124-34. doi: 10.1016/j.cbi.2016.06.007

16. Larasati RA, Harbuwono DS, Rahajeng E, Pradipta S, Nuraeni HS, Susilowati A, et al. The Role of Butyrate on Monocyte Migration and Inflammation Response in Patient With Type 2 Diabetes Mellitus. Biomedicines (2019) 7 (4):74. doi: 10.3390/biomedicines7040074

17. American Diabetes Association. 6. Glycemic Targets: Standards of Medical Care in Diabetes-2020. Diabetes Care (2020) 43(Supplement 1):S66-76. doi: $10.2337 / \mathrm{dc} 20-$ S006

18. Sircana A, Framarin L, Leone N, Berrutti M, Castellino F, Parente R, et al. Altered Gut Microbiota in Type 2 Diabetes: Just a Coincidence? Curr Diabetes Rep (2018) 18(10):98. doi: 10.1007/s11892-018-1057-6

19. Sharma S, Tripathi P. Gut Microbiome and Type 2 Diabetes: Where We Are and Where to Go? J Nutr Biochem (2019) 63:101-8. doi: 10.1016/ j.jnutbio.2018.10.003

20. Nishitsuji K, Xiao J, Nagatomo R, Umemoto H, Morimoto Y, Akatsu H, et al. Analysis of the Gut Microbiome and Plasma Short-Chain Fatty Acid Profiles in a Spontaneous Mouse Model of Metabolic Syndrome. Sci Rep (2017) 7 (1):15876. doi: 10.1038/s41598-017-16189-5

21. Liang H, Hussey SE, Sanchez-Avila A, Tantiwong P, Musi N. Effect of Lipopolysaccharide on Inflammation and Insulin Action in Human Muscle. PLoS One (2013) 8(5):e63983. doi: 10.1371/journal.pone.0063983

22. Trøseid M, Nestvold TK, Rudi K, Thoresen H, Nielsen EW, Lappegård KT. Plasma Lipopolysaccharide Is Closely Associated With Glycemic Control and Abdominal Obesity: Evidence From Bariatric Surgery. Diabetes Care (2013) 36(11):3627-32. doi: 10.2337/dc13-0451

23. den Besten G, van Eunen K, Groen AK, Venema K, Reijngoud DJ, Bakker BM. The Role of Short-Chain Fatty Acids in the Interplay Between Diet, Gut Microbiota, and Host Energy Metabolism. J Lipid Res (2013) 54(9):2325-40. doi: 10.1194/jlr.R036012

24. Deroover L, Boets E, Tie Y, Vandermeulen G, Verbeke K. Quantification of Plasma or Serum Short-Chain Fatty Acids: Choosing the Correct Blood Tube. J Nutr Health Food Sci (2017) 5(6):1-6. doi: 10.15226/jnhfs.2017.001112 
25. Fernandes J, Vogt J, Wolever TMS. Inulin Increases Short-Term Markers for Colonic Fermentation Similarly in Healthy and Hyperinsulinaemic Humans. Eur J Clin Nutr (2011) 65(12):1279-86. doi: 10.1038/ejcn.2011.116

26. Müller M, Hernández MAG, Goossens GH, Reijnders D, Holst JJ, Jocken JWE, et al. Circulating But Not Faecal Short-Chain Fatty Acids Are Related to Insulin Sensitivity, Lipolysis and GLP-1 Concentrations in Humans. Sci Rep (2019) 9(1):12515. doi: 10.1038/s41598-019-48775-0

27. Nancey S, Bienvenu J, Coffin B, Andre F, Descos L, Flourié B. Butyrate Strongly Inhibits In Vitro Stimulated Release of Cytokines in Blood. Dig Dis Sci (2002) 47(4):921-8. doi: 10.1023/A:1014781109498

28. Lachmandas E, van den Heuvel CNAM, Damen MSMA, Cleophas MCP, Netea MG, van Crevel R. Diabetes Mellitus and Increased Tuberculosis Susceptibility: The Role of Short-Chain Fatty Acids. J Diabetes Res (2016) 2016:6014631. doi: 10.1155/2016/6014631

29. Cleophas MCP, Ratter JM, Bekkering S, Quintin J, Schraa K, Stroes ES, et al. Effects of Oral Butyrate Supplementation on Inflammatory Potential of Circulating Peripheral Blood Mononuclear Cells in Healthy and Obese Males. Sci Rep (2019) 9(1):775. doi: 10.1038/s41598-018-37246-7

30. Magnusson MK, Isaksson S, Öhman L. The Anti-Inflammatory Immune Regulation Induced by Butyrate Is Impaired in Inflamed Intestinal Mucosa
From Patients With Ulcerative Colitis. Inflammation (2020) 43(2):507-17. doi: 10.1007/s10753-019-01133-8

Conflict of Interest: The authors declare that the research was conducted in the absence of any commercial or financial relationships that could be construed as a potential conflict of interest.

Publisher's Note: All claims expressed in this article are solely those of the authors and do not necessarily represent those of their affiliated organizations, or those of the publisher, the editors and the reviewers. Any product that may be evaluated in this article, or claim that may be made by its manufacturer, is not guaranteed or endorsed by the publisher.

Copyright (๑) 2021 Wibowo, Harbuwono, Tahapary, Kartika, Pradipta and Larasati. This is an open-access article distributed under the terms of the Creative Commons Attribution License (CC BY). The use, distribution or reproduction in other forums is permitted, provided the original author(s) and the copyright owner(s) are credited and that the original publication in this journal is cited, in accordance with accepted academic practice. No use, distribution or reproduction is permitted which does not comply with these terms. 